\title{
Warps and Accretion
}

\author{
Gyula I. G. Józsa ${ }^{1}$ \\ ${ }^{1}$ ASTRON (NWO) \\ Oude Hoogeveensedijk 4 \\ 7991 PD Dwingeloo \\ The Netherlands \\ email: jozsa@astron.nl
}

\begin{abstract}
Warps are a basic feature of disk galaxies. Usually they occur at radii where the optical disk fades and become most pronounced in the outermost gaseous disks.

As such, warps present a massive reservoir to replenish star forming material in the inner, star forming disks. Furthermore, some possible excitation mechanisms for warps connect their formation to the accretion of extragalctic material. Interactions or mergers with gas-rich companions or the direct accretion of the ambient intergalactic medium might lead to the formation of warps, at the same time supplementing fuel to maintain star formation in galactic disks.

Employing a number of $\mathrm{H}$ I studies of warped galaxies, including ultra-deep observations of the prototype warped galaxies NGC 5907 and NGC 4013, I discuss whether the observed kinematics may show evidence for a connection of warps and accretion from the ambient medium.
\end{abstract}

Keywords. galaxies: evolution, galaxies: kinematics and dynamics, galaxies: ISM, galaxies: formation, galaxies: intergalactic medium, galaxies: spiral, galaxies: structure

\section{Warps and accretion}

The formation and maintenance of warps form a long-standing problem for galaxy formation scenarios. Probably every galaxy shows a warp (Sancisi 1976, Sánchez-Saavedra et al. 1990, Reshetnikov \& Combes 1998, Reshetnikov \& Combes 1999, Schwarzkopf \& Dettmar 2001, Sánchez-Saavedra et al. 2003, Ann \& Park 2006). Traced out to the largest radii in the $\mathrm{H}$ I emission line, warps seem in most cases, while asymmetric, to be dominated by a grand-design S-shaped pattern (García-Ruiz et al. 2002).

Detailed studies of the structure of warps hint at the fact that warps are quite longlived Briggs 1990, Józsa 2007. However, theoretical attempts to describe warps in a self-contained manner, in closed-box models consisting of a disk and an optional (misaligned) Dark Matter halo, without the need of external agents, have failed to explain the observations so far or fail in follow-up studies adding additional complexities (LyndenBell 1965, Hunter \& Toomre 1969, Dekel \& Shlosman 1983, Sparke \& Casertano 1988, Kuijken 1991, Revaz \& Pfenninger 2004, Nelson \& Tremaine 1995, Dubinski \& Kuijken 1995). Hence, probably an external agent, apart from the Dark Matter halo and the baryonic disk component of a galaxy, is needed to maintain a warp over a sufficient time.

Following earlier suggestions (Ostriker \& Binney 1989, Binney 1992), more recent theoretical studies connect the warping phenomenon to the infall of matter into the potential well of spiral galaxies or the hierarchical growth of galaxies. External torques, as can be imposed on the disk by disrupted companion galaxies can excite a long-lived warp Shen \& Sellwood 2006, the warp material itself may consist of accreted material (van der Kruit 2007), and the long-lived internal misalignment of the Dark Matter halo as generated by cosmic infall may maintain a warp over the time scales of several dynamical 
times (several Gigayears, Bailin et al. 2005). Computer simulations turn out relatively long-lived warps by employing hydrodynamical simulations of cosmic infall, including satellite and gas accretion along filaments (Sánchez-Blázquez et al. 2009, Roškar et al. 2010).

In such a scenario, it is likely to expect that both mechanisms play a role, the accretion of (Dark) matter in a plane determined by the accretion flow, thus forming and maintaining a sub-structure in the potential which is torquing the disk, as well as the supply of fresh high-angular momentum gas onto the (outer) disk. Warps may thus form a reservoir of star forming material accreted with high angular momentum (van der Kruit 2007, Sancisi et al. 2008). Warps seem to be long-lived, but do not present a steady-state mode, hence their decomposition may lead to a gradual inflow of gas onto the inner disk through the loss of specific angular momentum as the angular momentum axes of inner and outer disk align. In this way, warps may act as a buffer for infalling cold gas and regulate the accretion of star forming material from the ambient medium onto the inner disk.

\section{Observational evidence for infall of matter in warped galaxies}

Dark Matter substructure. If the scenario outlined above is correct, and warps connect to infall in a preferred (misaligned) plane, then it is to be expected that not only gas is depleted on such a preferred plane, but also Dark Matter. The generation of (misaligned) sub-structure in the Dark Matter component through infall is expected in the standard picture of hierarchical structure formation (Bailin et al. 2005, Shen \& Sellwood 2006). Signatures of substructure may be found in detailed kinematical studies of warps. At the onset of a warp, the rotation curve should show a change, if the warped disk indeed traces a dynamically preferred plane. Indeed, Van der Kruit (2007) points out that often rotation curves show a sharp decline at the onset of the warp, while he explains this feature with a drop in the mass-surface density of the stellar disk. However, Józsa (2007) observes a distinct increase of the rotation velocity in the warped region in a sample of three disk galaxies, which certainly cannot be explained by a drop in the stellar surface-mass density. In M33, a similar increase at the onset of the warp is observed (Corbelli \& Schneider 1997). Roy (2010) simulates the structure of rotation curves under the influence of DM sub-structure as generated in a hierarchical model, and finds, that indeed significant changes of the measured rotation velocity is expected to occur. Hence, some rotation curves of warped galaxies show distinct signatures at the onset of warps that might be in favour of the hypothesis that Dark Matter substructure plays a role in the warped regions of galactic disks.

Anomalous gas. It has been pointed out that some galaxies exhibit an "anomalous" neutral gas component, significantly deviating in its kinematics from the net rotation of the galaxies (see Sancisi et al. 2008 for a summary, and Heald et al., this conference). Since their kinematics appear incompatible with a pure "fountain model" (Shapiro \& Field, the occurrence of such gaseous halos has been connected to the accretion of gas from the ambient intergalactic medium onto the disk. Fraternali \& Binney 2008 conclude that a contribution of low-angular momentum gas in addition to fountain material is needed to explain the kinematics of gaseous halos. In an accretion scenario, such low angular momentum material might comes from outer warped disks, formed by accretion, which supply gas to the inner disk in the process of their (slow) decomposition. Indeed, it has been remarked that anomalous gas is observed in warped disks. Józsa (2007) finds anomalous gas to be present in three galaxies selected to show a prototype symmetric warp. Certainly, these findings do not allow to draw a direct connection of warping 


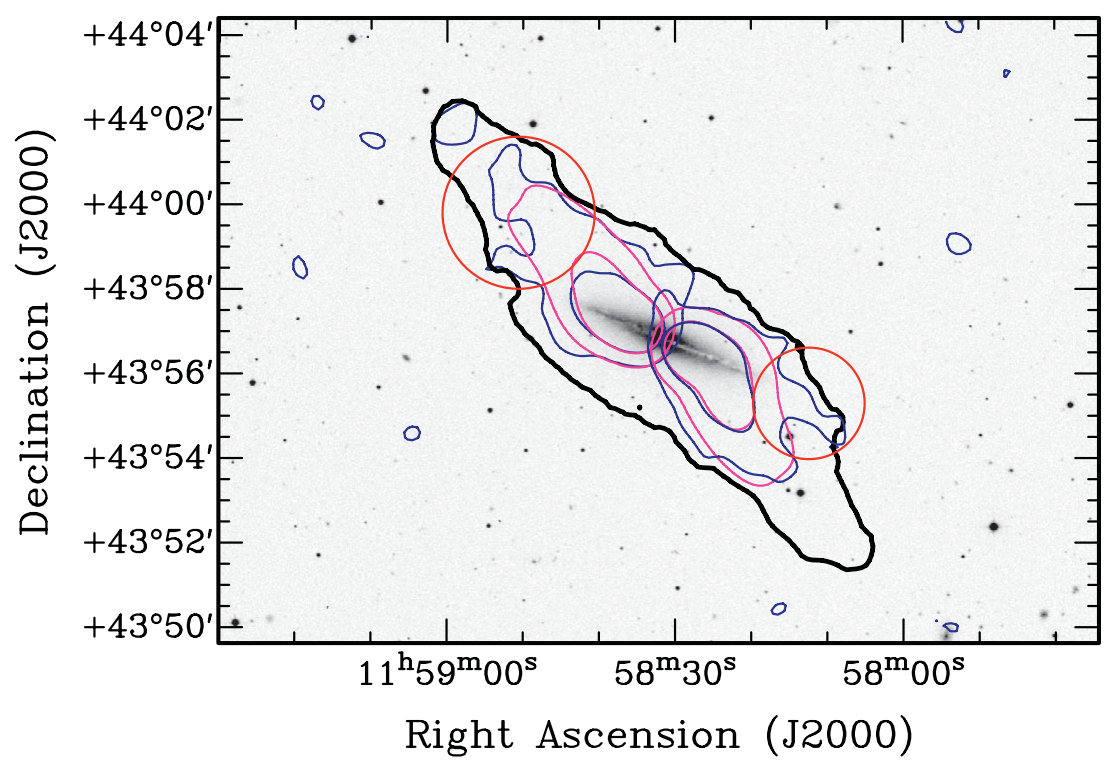

Figure 1. WSRT H I observations of NGC 4013. Black contours: H I total intensity map. Contour level: $5 \cdot 10^{18}$ atoms $\mathrm{cm}^{-2}$. Dark (blue) and light grey (pink) contours: two channel maps $\left(735 \mathrm{~km} \mathrm{~s}^{-1}\right.$ to the East and $924 \mathrm{~km} \mathrm{~s}^{-1}$ to the West). Light grey (pink): model data cube. Dark grey (blue): observation. Contours: $0.375,3.75 \mathrm{mJy} \mathrm{beam}^{-1}$. The grey (red) circles highlight the regions where the observation deviates from a flat-disk model.

properties of galaxies and the existence of anomalous gas. However, the systematic correlation of the presence of anomalous gas components in $\mathrm{H}$ I observations of galaxies with their warp properties will very soon become possible with the implementation of dedicated survey instruments for the observation of the $\mathrm{H}$ I emission line in galaxies.

To observe a possible accretion of high-angular momentum gas onto outer warped galaxy disks is the goal of an ongoing study with the Westerbork Synthesis Ratio Telescope (WSRT). The preliminary results of a deep survey of the two prototype warped galaxies NGC 4013 and NGC 5907, both observed with the WSRT for $17 \times 12 \mathrm{~h}$ shall be mentioned briefly. Former photometric studies revealed the presence of stellar streams around the galaxies, which were connected to the presence of the significant warps in the outskirts of the galaxies (Shang et al. 1997, Martínez-Delgado et al. 2008, MartínezDelgado et al. 2009). Our deep H I observations enable us to reveal anomalous gas components in both galaxies. In NGC 5907 anomalous gas is detected at the position of the cross-section of the stellar stream and the disk of NGC 5907, which might be stripped from the disrupted companion on a polar orbit. No extended gas component aligned with the stellar stream could be found. For NGC 4013 the situation might be slightly different. Comparing the observations with a model data cube (Fig.1), representing a best-fit model of a regularly rotating, warped disk, we note the presence of a slightly offset gas component that aligns with the stellar stream observed by Martínez-Delgado et al. (2009). While the NGC 5907-stellar stream system might resemble to some extent the interaction of the Milky Way with the Magellanic Cloud system, NGC 4013 matches the accretion scenario described above: a satellite galaxy is accreted on an orbit roughly aligned with the warped disk, and hence the alleged preferred plane, adding dark material and high angular momentum gas. 


\section{Summary}

Theory suggests that warps might be generated through the accretion of matter in the outskirts of galaxies. In that context, they might be important for the regulation of gas accretion in galaxies. Observations support this picture in the sense that in the few detailed H I studies, kinematical signatures in the gaseous components of the galaxies can be found that might support this picture. However, extended statistical studies as will be enabled with upcoming large-scale $\mathrm{H}$ I are necessary to draw firm conclusions.

\section{References}

Ann, H. B. \& Park, J.-C. 2006 New A, 11, 293

Bailin, J., Kawata, D., Gibson, B. K., et al. 2005, ApJ, 627, L17

Briggs, F. H. 1990, ApJ, 352, 15

Binney, J. 1992, ARA\&A, 30, 51

Corbelli, E. \& Schneider, S. E. 1997, ApJ, 479, 244

Dubinski, J. \& Kuijken, K. 1995, ApJ, 442, 492

Fraternali, F. \& Binney, J. J. 2008, MNRAS, 386, 395

García-Ruiz, I., Sancisi, R., \& Kuijken, K. 2002, A\& A, 394, 769

Hunter, C. \& Toomre, A. 1969, ApJ, 155, 747

Józsa, G. I. G. 2007, $A \mathscr{E} A, 468,903$

Kuijken, K. 1991, ApJ, 376, 467

Lynden-Bell, D. 1965, MNRAS, 129, 299

Martínez-Delgado, D., Pohlen, M.,Gabany, R. J., Majewski, S. R., Peñarrubia, J., \& Palma, C. 2009, ApJ, 692, 955

Martínez-Delgado, D., Peñarrubia, J., Gabany, R. J., Trujillo, I., Majewski, S. R., \& Pohlen, M. 2008, ApJ, 689, 184

Nelson, R. W. \& Tremaine, S. 1995, MNRAS, 275, 897

Ostriker, E. C. \& Binney, J. J. 1989, MNRAS, 237, 785

Reshetnikov, V.\& Combes, F. 1998, A\&A A, 337, 9

Reshetnikov, V. \& Combes, F. 1999, A\&AS, 138, 101

Roškar, R., Debattista, V. P., Brooks, A. M., Quinn, T. R., Brook, C. B., Governato, F., Dalcanton, J. J., \& Wadsley, J. 2010, MNRAS, 408, 783

Sánchez-Blázquez, P., Courty, S., Gibson, B. K., \& Brook, C. B. 2009, MNRAS, 398, 591

Shapiro, P. R. \& Field, G. B. 1976, ApJ, 205, 762

Sánchez-Saavedra, M. L., Battaner, E., \& Florido, E. 1990, MNRAS, 246, 458

Sánchez-Saavedra, M. L., Battaner, E., Guijarro, A., López-Corredoira, M., \& Castro-Rodríguez, N. 2003, $A \mathscr{E} A, 399,457$

Sancisi, R. 1976, A\& A, 53, 159

Sancisi, R., Fraternali, F., Oosterloo, T., \& van der Hulst, T. 2008, A\&AA Rev., 15, 189

Shen, J. \& Sellwood, J. A. 2006, MNRAS, 370, 2

Schwarzkopf, U. \& Dettmar, R.-J. 2001, A\& A, 373, 402

Sparke, L. S. \& Casertano, S. 1988, MNRAS, 234, 873

Revaz, Y. \& Pfenniger, D. 2004, A\&A, 425, 67

Roy, N. 2010, ApJ, 723, 781

Shang, Z., Brinks, E., Zheng, Z., et al. 1998, ApJ, 504, L23

van der Kruit, P. C. 2007, $A \& A, 466,883$ 